The University of Southern Mississippi

The Aquila Digital Community

Faculty Publications

$9-1-2006$

\title{
Introduction: Self-Organization In Nonequilibrium Chemical Systems
}

Irving R. Epstein

Brandeis University

John A. Pojman

University of Southern Mississippi

Oliver Steinbock

Florida State University

Follow this and additional works at: https://aquila.usm.edu/fac_pubs

Part of the Chemistry Commons

\section{Recommended Citation}

Epstein, I. R., Pojman, J. A., Steinbock, O. (2006). Introduction: Self-Organization In Nonequilibrium Chemical Systems. Chaos, 16(3).

Available at: https://aquila.usm.edu/fac_pubs/2266

This Article is brought to you for free and open access by The Aquila Digital Community. It has been accepted for inclusion in Faculty Publications by an authorized administrator of The Aquila Digital Community. For more information, please contact Joshua.Cromwell@usm.edu. 


\title{
Introduction: Self-organization in nonequilibrium chemical systems
}

\author{
Irving R. Epstein \\ Department of Chemistry, MS 015, Brandeis University, Waltham, Massachusetts 02454-9110 \\ John A. Pojman \\ Department of Chemistry and Biochemistry, The University of Southern Mississippi, Hattiesburg, \\ Mississippi 39406-0001 \\ Oliver Steinbock \\ Department of Chemistry and Biochemistry, Florida State University, Tallahassee, Florida 32306-4390
}

(Received 23 August 2006; accepted 23 August 2006; published online 27 September 2006)

\begin{abstract}
The field of self-organization in nonequilibrium chemical systems comprises the study of dynamical phenomena in chemically reacting systems far from equilibrium. Systematic exploration of this area began with investigations of the temporal behavior of the Belousov-Zhabotinsky oscillating reaction, discovered accidentally in the former Soviet Union in the 1950s. The field soon advanced into chemical waves in excitable media and propagating fronts. With the systematic design of oscillating reactions in the 1980s and the discovery of Turing patterns in the 1990s, the scope of these studies expanded dramatically. The articles in this Focus Issue provide an overview of the development and current state of the field. (C) 2006 American Institute of Physics. [DOI: 10.1063/1.2354477]
\end{abstract}

\section{INTRODUCTION}

Readers of this journal are well versed in nonlinear dynamics. In this Focus Issue we highlight the dynamics of chemically reacting systems far from equilibrium with emphasis on such systems' ability to self-organize. Nonlinear chemical dynamics is concerned with oscillations in wellstirred systems, chemical waves and fronts, chaos, and stationary spatial patterns. Other focus issues have overlapped the field: control of chaos [Chaos 7 (4), 1997], understanding ventricular fibrillation [Chaos 8 (1), 1998], nonlinear science in chemical engineering [Chaos 9 (1), 1999], and nonlinear dynamics in polymeric systems [Chaos 9 (2), 1999].

Chemists are perhaps more surprised by the spectacular, almost magical pattern formation that can arise in nonequilibrium systems than are physicists or engineers. This is probably the result of two factors. First, chemists focus on molecules. Such emphasis on the molecular level makes it difficult to envision how macroscopic patterns can form. Second, much of what chemists learn and study stresses the equilibrium state. Quite often, even diffusion is not addressed in the undergraduate curriculum and so chemists are ill prepared to consider nonequilibrium systems.

We seek here to provide an overview of the field of nonlinear chemical dynamics, though not by any means a comprehensive review. Readers are referred to other works for more information. ${ }^{1}$ We also must confess to neglecting a very important area, viz., the application of these ideas to biological and biochemical systems. ${ }^{2}$

\section{HISTORY OF THE FIELD}

Fechner described an electrochemical cell that produced an oscillating current, the first published report of oscillations in a chemical system, in $1828 .^{3}$ Ostwald reported in 1899 that the rate of chromium dissolution in acid periodi- cally increased and decreased. ${ }^{4}$ Because both systems are inhomogeneous, it was believed then, and through much of the next century, that homogeneous oscillating reactions were impossible.

The first homogeneous isothermal chemical oscillator to be described was the reaction of iodate, iodine, and hydrogen peroxide, studied by William C. Bray, and later by his student Herman Liebhafsky. Hydrogen peroxide decomposes to oxygen and water. ${ }^{5}$ The rate of evolution of oxygen and the $\mathrm{I}_{2}$ concentration were found to vary nearly periodically. However, for the next 50 years, chemists would believe that the reaction was not really homogeneous and that the oscillations were an artifact of dust or bubbles. Noyes and coworkers revived the work of Bray and Liebhafsky in the 1970s and succeeded in convincing the chemical community that the Bray reaction represented a genuine chemical oscillator. $^{6}$

The beginning of modern nonlinear chemical dynamics can be traced to Boris Pavlovich Belousov, who was looking for an inorganic analog of the Krebs cycle, a key metabolic process in which citric acid is an intermediate. In 1950 he studied a solution of bromate and citric acid in sulfuric acid with ceric ions $\left(\mathrm{Ce}^{+4}\right)$. He expected to see the monotonic conversion of yellow $\mathrm{Ce}^{+4}$ into colorless $\mathrm{Ce}^{+3}$. Instead, the solution became clear and then became yellow again, over and over! Belousov also noted that, unstirred in a graduated cylinder, the solution exhibited traveling waves of yellow. He submitted a manuscript in 1951, but it was rejected. ${ }^{7} \mathrm{He}$ labored six more years and submitted a revised manuscript to another journal, but that editor insisted that the paper be shortened to a letter before further consideration. Belousov gave up on publishing his work, but he kept his manuscript, which circulated among colleagues in Moscow. His only publication on this reaction appears in the unrefereed abstracts of a conference on radiation biology. ${ }^{8}$ 
In 1961, Anatol Zhabotinsky, a graduate student in biophysics at Moscow State University, began looking at the same system with malonic acid and obtained a better formulation, which did not produce precipitate. At least ten papers on the Belousov-Zhabotinsky (BZ) reaction were published in Russian before the first one in English. ${ }^{9}$ A conference was held in Prague in 1968 on Biological and Biochemical Oscillators, where Zhabotinsky presented some of his results. This meeting motivated many in the Eastern Bloc to study the $\mathrm{BZ}$ reaction, and the publication of the proceedings in English brought the BZ reaction to the attention of several Western chemists as well. ${ }^{10}$

Belousov used the redox indicator ferroin in some experiments to heighten the color change during oscillations. Ferroin is red in reduced solution and blue in oxidized form, providing a more easily visible variation than the pale yellow to colorless change of the ceric-cerous system. Zaikin and Zhabotinsky found that ferroin alone could catalyze the BZ reaction without cerium. ${ }^{11}$ This advance allowed them to study unstirred solutions in thin layers, in which they discovered propagating chemical waves. Now a homogeneous system was shown to exhibit not only temporal but also spatial self-organization.

In 1980 the Lenin Prize was awarded to Belousov, who had died in 1970, and A. M. Zhabotinsky, V. I. Krinsky, and G. R. Ivanitsky for their work on the BZ reaction.

Before, and even during, the development of the BZ reaction, a number of papers were being written in the west on why true homogeneous oscillating reactions were impossible. An incorrect understanding of the Second Law of Thermodynamics motivated these objections. The entropy of the universe must increase during a spontaneous process. Chemists prefer to focus on the Gibbs Free Energy, $G=H-T S$. For an isolated system this must approach a global minimum during a spontaneous chemical reaction. What many chemists mistakenly assumed about reactions like the BZ reaction was that reactants were being converted to products and then products back to reactants. This would have required the free energy to decrease and then increase-a clear violation of the Second Law. This erroneous understanding may have arisen from thinking of a chemical oscillator as analogous to a pendulum.

A chemical oscillator is fundamentally different from a pendulum. When a chemical reaction oscillates, it never passes through its equilibrium point. Instead, chemical oscillation is a far-from-equilibrium phenomenon, governed by the laws of nonequilibrium thermodynamics. ${ }^{12}$ Beginning in the 1930s, Lars Onsager, Ilya Prigogine and others realized that thermodynamics could be applied to systems far from equilibrium, but that a new theory was required. Prigogine and co-workers in Brussels focused on chemical systems, pointing out that a system could organize (decrease its entropy), so long as the net entropy change in the universe was positive. ${ }^{13-15}$ For example, the concentrations of the intermediates in a reaction can oscillate while the free energy monotonically decreases as a result of the continuing conversion of high free energy reactants into low free energy products. Any decrease in entropy caused by the periodic concentra- tion changes is more than compensated by an entropy increase from the other processes.

Prigogine pointed out in 1955 that open systems, i.e., systems open to the exchange of matter and/or energy with their surroundings, kept far from equilibrium could exhibit spontaneous self-organization by dissipating energy to the surroundings to compensate for the entropy decrease in the system. ${ }^{13}$ He called the temporal or spatial structures that can arise in this way, dissipative structures. A closed system must reach equilibrium and so can exhibit only transitory oscillations as it approaches equilibrium. Sustained oscillations require an open system with a continuous flow of new reagents and removal of waste products.

Another impediment to the acceptance of oscillating reactions was the lack of a chemically plausible mechanism. The first such model was proposed by Prigogine and Lefever in $1968^{16}$ and dubbed the "Brusselator" by Tyson in $1973 .{ }^{17}$

In 1977, Nicolis and Prigogine summarized the work of the Brussels school in a book entitled Self-Organization in Nonequilibrium Systems. ${ }^{15}$ For his work on nonequilibrium systems, Ilya Prigogine was awarded the 1977 Nobel Prize in Chemistry.

\section{The Belousov-Zhabotinsky reaction}

A chemist wants to develop a molecular-level "map" or mechanism of each chemical reaction. A mechanism is a series of elementary reactions that involve actual molecular collisions that lead to transformation. This is an extremely difficult task for all but the simplest of reactions.

Zhabotinsky and co-workers had made significant progress in understanding the mechanism of the $\mathrm{BZ}$ reaction, but their work was largely unknown in the west. The first publications in English to recognize the work of Belousov and Zhabotinsky were by the Danish scientist Hans Degn. ${ }^{18}$ He studied some aspects of the BZ reaction, but he was unable to propose a complete mechanism for the system. The Prague conference in 1968 afforded an opportunity for Zhabotinsky and some of the other Russian scientists to present their work and meet some of the more intrepid westerners.

Field, Körös, and Noyes learned of the BZ reaction and after careful study were able to explain the qualitative behavior of the $\mathrm{BZ}$ reaction using the same principles of chemical kinetics and thermodynamics that govern "ordinary" chemical reactions. They published their mechanism, now known as the FKN mechanism, in a classic paper in $1972 .{ }^{19}$ A quantitative numerical simulation of the oscillatory behavior was published a few years later. ${ }^{20}$

Field and Noyes managed to simplify the FKN mechanism. They obtained a model that had only three variable concentrations yet maintained all the essential features of the full BZ reaction. The model was dubbed the "Oregonator."21

\section{Systematic design}

By the late 1970s many chemists were aware of oscillating reactions and agreed that they were authentic chemical phenomena. There were, of course, many biological oscilla- 
tors known, but efforts to define a set of necessary and sufficient conditions for a chemical reaction to oscillate proved fruitless.

In the mid-1970s, two efforts began that were to converge toward a systematic approach to building chemical oscillators. Epstein and Kustin at Brandeis University identified several autocatalytic inorganic reactions that they thought could be turned into new chemical oscillators. Meanwhile, at the Paul Pascal Research Center in Bordeaux, a group of scientists led by Adolphe Pacault had pioneered the use of the continuous flow stirred tank reactor (CSTR), a tool familiar to chemical engineers but essentially unknown to chemists, to provide an open system suited to the study of oscillating reactions. ${ }^{22}$ Two members of the Bordeaux group, Patrick De Kepper and Jacques Boissonade, developed an abstract model that suggested how oscillations might be obtained in a CSTR by perturbing a bistable chemical system. ${ }^{23}$ De Kepper went to Brandeis in late 1979, and within a few months the team had developed the first systematically designed oscillating reaction, the arsenite-iodate-chlorite system. ${ }^{24}$ The technique was refined and used to develop dozens of new oscillators over the next decade.

A topic that created great interest among physicists and mathematicians in the 1970s and 1980s is deterministic chaos. A chaotic system is unpredictable, but not random. Deterministic equations describe the system, but the system is so sensitive to its initial conditions that its future behavior is inherently unpredictable beyond some relatively short period of time. Schmitz and Hudson reported in 1977 that the BZ system behaves chaotically under certain conditions in a flow reactor. ${ }^{25}$ Further studies in the 1980s and 1990s have confirmed the existence of chaos in chemical systems and have shed new light on its origins. ${ }^{26}$

\section{CHEMICAL WAVES}

A surprisingly understudied but nonetheless important field of physical chemistry concerns the spatial coupling of local reaction processes. This coupling can result from a variety of different transport phenomena such as diffusion, convection, and electromigration. Often, several types of spatial coupling are present in a given experimental system, but most investigations are dedicated to examples in which one specific form of transport is dominant. In chemistry, research on such spatially extended systems is often motivated by its relevance to industrial processes in large reactor systems and also by its potential to provide simple models that mimic complex phenomena in living systems, such as nerve conduction as well as intra- and extracellular communication. Moreover, chemists hope to identify powerful new synthesis strategies and control the nano- and microscale assembly of advanced materials. These are, however, long-term goals, and only a small number of technological applications have actually been developed so far.

A classic example of spatial coupling of local reaction processes is the large class of reaction-diffusion systems. These systems are typically modeled by appropriate sets of coupled partial differential equations that describe the spatiotemporal concentration evolution of all, or at least the dynamically most relevant, chemical species. Typically, the transport terms obey simple Fickian diffusion and the kinetic (reaction) terms show mild nonlinearities such as encountered in the aforementioned Belousov-Zhabotinsky or BrayLiebhafsky reactions. An interesting and seemingly simple behavior that can result from such a constellation is front propagation into unstable states. The chlorite-tetrathionate (CT) reaction, for example, can show front propagation and has been studied by several groups in the last decades. ${ }^{27}$ The acid autocatalytic front in this system can be readily monitored by employing appropriate $p \mathrm{H}$ indicators. Such experiments reveal constant front profiles and constant front velocities of the order of millimeters per minute. Tóth et al. showed that the effective diffusion coefficient of the autocatalyst, $\mathrm{H}^{+}$, can be decreased by employing polymer gels in which immobilized carboxylate groups reversibly bind hydrogen ion. ${ }^{28}$ The resulting contrast between the diffusion coefficients of the key players, hydrogen ion and tetrathionate ion, induces a, less obvious, long-wavelength instability. This instability manifests itself as nonplanar, nonstationary fronts. The resulting dynamics of the reaction front are very similar to those found in the Kuramoto-Shivashinsky equation, ${ }^{29}$ and also share features with growth patterns in bacterial colonies. ${ }^{30}$

Front propagation in the CT reaction shows additional features that are continuing to attract the interest of chemists in this field. Like many similar systems (e.g., the iodatearsenous acid reaction ${ }^{31}$ ) the $\mathrm{CT}$ reaction can show striking hydrodynamic flows that are intimately related to the concentration changes within the traveling front. ${ }^{32}$ More precisely, downward moving fronts can become buoyantly unstable due to the stratification of dense product solution on top of the lighter reactant solution. This scenario results in rather spectacular density fingering. ${ }^{33}$ Similar complications are also encountered in the synthesis of certain polymers via frontal polymerization. In the latter type of systems, we also encounter nonisothermal conditions, as the reactions involved are often highly exothermic. These factors can induce additional instabilities such as the intriguing spin modes that were studied by Pojman et al. ${ }^{34}$

There are numerous other topics related to propagating fronts and their instabilities, but their discussion would go beyond the scope of this Introduction. However, it is worth mentioning that there is continued research activity aimed at perturbing and controlling front propagation via electric ${ }^{35}$ and magnetic ${ }^{36}$ fields as well as other externally imposed stimuli. ${ }^{37}$ Moreover, ideas are being pursued to utilize chemical front propagation as a tool to solve optimization problems in an unconventional, i.e., noncomputer based, and highly parallel fashion. For instance, Steinbock et al. used $\mathrm{BZ}$ fronts to demonstrate the analysis of shortest paths in labyrinths $^{38}$ and the construction of chemical logic gates. ${ }^{39}$ Related ideas are also explored by groups around Yoshikawa $^{40}$ and Gorecki. ${ }^{41}$ Lastly, we emphasize that chemical front propagation is also observed in nonsolution based reaction-diffusion media as well as in a great variety of electrochemical systems. Examples include catalytic surface reactions, ${ }^{42}$ electrodeposition phenomena such as the "electroless" deposition of copper, ${ }^{43}$ and front propagation during metastable pitting on steel. ${ }^{44}$ 
The previous discussion shows a great diversity in the types of experimental systems that can sustain traveling fronts. Perhaps not surprisingly, the same holds for the phenomenon of wave propagation. Obviously not all frontsupporting reactions can recover a state that allows additional fronts to move through medium, but the list of examples is still long and diverse. Once again, we find important examples in the field of electrochemistry with the classic one being the dissolution of iron in nitric acid. ${ }^{45} \mathrm{Un}-$ der appropriate conditions, the metal is in a passive state that is caused by a thin, spontaneously forming oxide layer on its surface. Local perturbations of this protective film can trigger a corrosion pulse that travels at relatively high speeds (up to $1 \mathrm{~m} / \mathrm{s}$ ) over the metal surface. In its wake, however, the metal reforms the protective oxide layer, thus re-establishing its original excitable state. Similar wave behavior is observed in many aqueous autocatalytic reactions. In the latter cases, the underlying mechanisms are often of a pure reactiondiffusion type. Over the last three decades, the BZ reaction has become the most frequently studied model among excitable reaction-diffusion media.

In the BZ reaction and similar systems, waves are typically studied to obtain new insights into the dynamics of complex patterns such as rotating spirals and chemical turbulence. Consequently, most investigations have focused on quasi-two-dimensional media and, for technical reasons, to a lesser extent on three-dimensional systems. A significantly smaller number of studies have explored pseudo-onedimensional BZ media. This specific spatial constraint can be approximated in an annular gel reactor. Already in 1987, Noszticzius et al. constructed and demonstrated such a device that allows the experimentalist to probe $\mathrm{BZ}$ waves under nontransient conditions. ${ }^{46}$ Although these waves are externally sustained and hence exist for only as long as the reactor pumps are operated, this type of ring reactor has rather complex boundary conditions. Phenomena that require shorter detection times are therefore more appropriately studied in closed systems such as small-diameter glass capillaries. However, special care has to be taken to avoid the formation of hydrodynamic flow patterns that could induce various artifacts.

Of foremost interest regarding excitation waves in 1D is the wavelength-velocity dependence of (ideally infinite) wave trains. This dependence is often referred to as the system's dispersion relation. The classical $\mathrm{BZ}$ reaction seems to have large parameter regions in which the dispersion relation is monotonically increasing while saturating at the velocity of the solitary pulse. This feature is called "normal dispersion" and stems from the refractory zone in the wake of each pulse. Within this zone, the system recovers its original excitability by adjusting the concentration levels of certain control species. In the BZ reaction, the inhibitory bromide ion acts as the main control species. ${ }^{47}$ Moreover, there is an absolute refractory zone close to the pulse where no new excitation cycle can be induced. The absolute refractory zone causes the directionality of wave propagation and is also responsible for the lack of interference and reflection phenomena that is observed for typical excitation waves. In recent years, anomalous dispersion relations have been observed and studied by several groups. These anomalies include anomalous slope, gaps, finite band widths, and bistability and are referenced in the article by Manz and Steinbock in this Focus Issue.

\section{PATTERN FORMATION}

Already around the end of the nineteenth century, scattered reports began to document the existence of spatial and spatio-temporal patterns in the realm of chemistry and its subdisciplines. Classic examples include precipitation patterns such as Liesegang rings and related geochemical patterns including band structures in agates. ${ }^{48}$ Also in electrochemistry and metallurgy, surprising spiral structures were observed in the electro-codeposition of silver-antimony alloys. ${ }^{49}$ However, the time was not ripe for systematic investigations of these phenomena, partly because our modern understanding of nonequilibrium thermodynamics had not yet been developed. Regarding experimental studies of chemical pattern formation in dissipative systems, the modern era started in the 1970s with the pioneering work of a small number of scientists, most prominently Zhabotinsky, Zaikin, and Winfree. ${ }^{50}$ Simple but astonishing experiments employing thin layers of the BZ solution were published that showed spatio-temporal structures such as target patterns and rotating spiral waves. Today, basic research on these patterns continues and more and more experimental examples document their existence in a broad range of physical, chemical, and biological systems.

Another central figure in the development of nonlinear chemical dynamics is the well-known mathematician Alan Turing. In 1952, Turing published a landmark paper entitled "The Chemical Basis of Morphogenesis." ${ }^{, 51}$ This paper predicted the existence of stationary concentration patterns in autocatalytic reaction-diffusion systems. However, the first experimental examples of these Turing patterns were reported only in 1990. The latter work by groups around Patrick De Kepper ${ }^{52}$ and Harry Swinney ${ }^{53}$ employed the chlorite-iodide-malonic acid (CIMA) reaction in a continuously-fed unstirred gel reactor. These stationary nonequilibrium structures are typically spots on a hexagonal lattice or stripe patterns with submillimeter-scale wavelengths.

In subsequent investigations of traveling and stationary patterns, it became clear that tools for the external perturbation and manipulation of the structures had the potential to open up new realms of investigation. Here, we want to highlight and briefly discuss two specific examples, namely the external control of photosensitive reaction media and the use of soft-lithography for the production of microscale reactor arrays. Visible light has been utilized as a tool to control and modulate the formation of Turing patterns. For example, Dolnik et al. ${ }^{54}$ studied the response of hexagonal Turing structures with pattern defects to spatially patterned visible light. Specifically, they found that defects are removed most effectively if the wavelength of the external forcing is slightly larger than the natural wavelength of the pattern. Other interesting applications concern traveling waves in the ruthenium-catalyzed BZ reaction. This system can be inhibited by blue light (maximal effect around $454 \mathrm{~nm}$ ) that 
promotes the production of the inhibitor species bromide. ${ }^{55}$ Kuhnert et al. used this feature to perform simple imageprocessing tasks. ${ }^{56}$ Steinbock et al. developed tools that allow for the controlled production, destruction and placement of spiral waves using localized perturbations created by an argon-laser beam. ${ }^{57}$ Showalter and co-workers developed and used intricate spatio-temporal feedback and control algorithms to study wave dynamics in subexcitable media, complex networks, and swarming. ${ }^{58}$ Lastly, the photosensitivity of the ruthenium-catalyzed BZ reaction can also be exploited for spatially extended, oscillatory media. For example, Petrov et al. ${ }^{59}$ investigated resonant forcing and Lin et al. Bloch-front turbulence. ${ }^{60}$

Although immensely versatile, light-induced photochemical perturbations of pattern forming systems fail to manipulate the dynamics at small length scales. This limitation is mainly due to diffusion of the inhibitory species produced in the illuminated region. Similar limitations exist for, otherwise quite powerful, techniques in which the catalyst is patterned and immobilized on membranes using inkjet-printer technology. ${ }^{61}$ To investigate more intricate spatial constraints, microreactor arrays can be produced using various types of lithography. These constraints are obviously static and cannot be easily altered in time. In the context of pattern-forming, catalytic, surface reactions, lithography has been used to create microcomposite systems in which front nucleation can be controlled. Specific examples include $\mathrm{Pt}(110)$ surfaces with lithographically controlled $\mathrm{Ti}$ or $\mathrm{Rh}$ overlayers. ${ }^{62}$ For the BZ reaction, Ginn et al. explored the use of soft lithography to control wave propagation through channel networks as narrow as $50 \mu \mathrm{m} .{ }^{63}$ They also investigated the dynamics of spiral wave rotation in grid-like networks of excitable channels and reported sequences of complex tip trajectories. ${ }^{64}$

Pattern-forming systems with even smaller reactor units have been developed and studied at Brandeis University. Vanag, Epstein and co-workers have investigated the BZ reaction in microemulsions consisting of small aqueous BZ droplets surrounded by a monolayer of the surfactant AOT in a continuous oil phase. ${ }^{65}$ By varying the relative amounts of oil, water, and surfactant, they can control the size of and separation between the droplet-reactors. They have found a variety of new patterns, such as inwardly rotating spirals ("antispirals") 66 and segmented traveling waves ("dashed waves"), ${ }^{67}$ not previously observed in simpler reactiondiffusion systems. A comparison between the length scales of the micelles $(5-20 \mathrm{~nm})$ in the BZ-AOT system and the structural features currently produced by lithography $(10-50 \mu \mathrm{m})$ reveals a remarkable gap of three orders of magnitude. We believe that this gap hides interesting new phenomena. Especially, we see a need to explore the behavior of wave patterns at this threshold between macroscopic heterogeneous and quasihomogeneous but anisotropic media.

The various facets of modern research on chemical pattern formation involve many other intriguing and equally important research topics. Our short introduction has failed so far to even mention current activities aiming to understand and control spiral-defect chaos, three-dimensional wave patterns, instabilities in real-world fuel cells and catalytic de- vices. Also, an uncounted number of mysteries remain unanswered regarding the role of chemical waves and patterns in living organisms. We hope that this short and modest introduction has set the stage for the research articles in this special issue and spiked the curiosity of the reader.

\section{THIS ISSUE}

This issue aims to provide an incomplete but representative cross section of some of the exciting research in the field of self-organization in chemical systems. In the beginning of our discussion of pattern formation in chemical systems, we discussed the classic example of Liesegang rings that are based on precipitation dynamics in cross-gradient systems. In recent years, Grzybowski et al. have used similar scenarios for constructing nonlinear chemical microsystems. Here, they review various experimental methodologies that allow for the production of unconventional chemical sensors and amplifiers.

Taylor and Britton investigate the impact of heterogeneities on three-dimensional wave propagation in a reactiondiffusion system with and without advection. The experimental part of this study employs magnetic resonance imaging to probe the behavior within optically opaque, porous media. Three-dimensional wave phenomena are also discussed by Yamaguchi et al. However, the latter authors investigate intricate vortex patterns in homogeneous reaction media using optical techniques.

A technologically important problem is discussed by Mikhailov et al. The authors investigate the onset of localized corrosion on metal surfaces. Specifically they analyze metastable pitting in the framework of stochastic temporal and spatiotemporal models.

Chemical wave propagation and in particular instabilities of wave trains are discussed by Manz and Steinbock. This contribution features experiments with the cyclohexanedione-BZ reaction in a pseudo-one-dimensional setting. Foremost, the authors present experimental evidence for breathing and backfiring pulses. Nonlinear dynamics in electrochemical systems is also studied by Rivera et al. Using numerical simulations, the authors characterize synchronization phenomena for oscillator pairs with unidirectional and bidirectional coupling. Wave propagation can also be observed in experimental systems that are profoundly different from autocatalytic reaction media. Shibata and Mikhailov present a theoretical study of Langmuir monolayers containing chiral molecules that induce phenomena such as target patterns and spiral waves.

Rohlf et al. report results of numerical simulations concerning spiral wave rotation in spherical geometries of various thicknesses. Their findings reveal interesting similarities between 2D and 3D media and could also be of relevance for improving our understanding of excitation dynamics in heart muscles. Marts et al. discuss chemical dynamics observed in response to a different class of external constraints. In their study, a pseudo-two-dimensional, oscillatory reaction medium is exposed to spatially homogeneous, oscillatory forcing. Their experiments employ the photosensitive BZ reaction and are complemented by theoretical studies. 
The group of Nakato studied electrochemical oscillations of $p$-type $\mathrm{Si}(111)$ immersed in $\mathrm{HF} / \mathrm{CuSO}_{4}$ solution. Their article describes surface changes during periodic and chaotic oscillations that are a crucial component of the reaction mechanism proposed in this work. The paper by Marek et al. investigates nonlinear chemical dynamics in the context of catalytic mufflers, thus exemplifying the importance of this field of research for technologically relevant problems.

Benyaich $e t$ al. present theoretical results regarding the spatio-temporal behavior in open gel reactors. Their results, obtained for the iodate-arsenous acid reaction, reveal a multiplicity of inhomogeneous stationary solutions. Boissonade et al. discuss the origin of "spatial bistability" in gel reactors. In addition, the authors present experimental results on chemo-mechanical coupling and spectacular wave-induced changes in the size and shape of gel systems.

Turing patterns are subject of the study by Yang et al. However, the authors go beyond the more familiar hexagonal and striped patterns and investigate superlattice Turing structures using a mix of experiments and numerical simulations.

In a rather different arena of study, Asakura et al. provide experimental results and analysis for considering chiral symmetry breaking in a propagating front of an organic crystallization.

\section{ACKNOWLEDGMENTS}

O.S. acknowledges support from the NSF Grant No. CHE-0513912. I.R.E. was supported by NSF Grant No. CHE-0615507.

${ }^{1}$ P. Gray and S. K. Scott, Chemical Oscillations and Instabilities (Clarendon, Oxford, 1990); S. K. Scott, Oscillations, Waves and Chaos in Chemical Kinetics (Oxford University Press, Oxford, 1994); R. Kapral and K. Showalter (Kluwer Academic, Dordrecht, 1995); I. R. Epstein and K. Showalter, J. Phys. Chem. 100, 13132 (1996); I. R. Epstein and J. A. Pojman, An Introduction to Nonlinear Chemical Dynamics: Oscillations, Waves, Patterns and Chaos (Oxford University Press, New York, 1998).

${ }^{2}$ A. Goldbeter, Rhythms and Chaos in Biochemical and Cellular Systems (Cambridge University Press, Cambridge, 1995).

${ }^{3}$ A. T. Fechner, Schweigg. J. 53, 61 (1828).

${ }^{4}$ W. Ostwald, Phys. Z. 8, 87 (1899).

${ }^{5}$ W. C. Bray, J. Am. Chem. Soc. 43, 1262 (1921); W. C. Bray and H. A. Liebhafsky, J. Phys. Chem. 53, 38 (1931).

${ }^{6}$ K. R. Sharma and R. M. Noyes, J. Am. Chem. Soc. 91, 202 (1975)

${ }^{7}$ A. T. Winfree, J. Chem. Educ. 61, 661 (1984).

${ }^{8}$ B. P. Belousov, Sbornik Referatov po Radiatsionni Meditsine, 145 (1958). ${ }^{9}$ H. Degn, Nature (London) 213, 589 (1967); H. Degn and D. E. F. Harrison, J. Theor. Biol. 22, 238 (1969).

${ }^{10}$ B. Chance, E. K. Pye, A. K. Ghosh, and B. Hess, Biological and Biochemical Oscillators (Academic, New York, 1973).

${ }^{11}$ A. N. Zaikin and A. M. Zhabotinsky, Nature (London) 225, 535 (1970).

${ }^{12}$ S. R. de Groot and P. Mazur, Nonequilibrium Thermodynamics (Dover, New York, 1984).

${ }^{13}$ I. Prigogine, Thermodynamics of Irreversible Processes (Wiley, New York, 1955).

${ }^{14}$ I. Prigogine, Non-Equilibrium Statistical Mechanics (Interscience, New York, 1962); P. Glansdorff and I. Prigogine, Thermodynamics of Structure, Stability and Fluctuations (Wiley, New York, 1971); I. Prigogine, From Being to Becoming: Time and Complexity in the Physical Sciences (Freeman, New York, 1980).

${ }^{15}$ G. Nicolis and I. Prigogine, Self-Organization in Nonequilibrium Systems (Wiley, New York, 1977).

${ }^{16}$ I. Prigogine and R. Lefever, J. Chem. Phys. 48, 1695 (1968).

${ }^{17}$ J. Tyson, J. Chem. Phys. 58, 3919 (1973).

${ }^{18}$ P. Lindblad and H. Degn, Acta Chem. Scand. (1947-1973) 21, 791 (1967); H. Degn, ibid. 21, 1057 (1967).
${ }^{19}$ R. M. Noyes, R. J. Field, and E. Körös, J. Am. Chem. Soc. 94, 1394 (1972); R. J. Field, E. Körös, and R. M. Noyes, ibid. 94, 8649 (1972).

${ }^{20}$ D. Edelson, R. J. Field, and R. M. Noyes, Int. J. Chem. Kinet. 7, 417 (1975).

${ }^{21}$ R. J. Field and R. Noyes, J. Chem. Phys. 60, 1877 (1974).

${ }^{22}$ A. Pacault, P. Hanusse, P. De Kepper, C. Vidal, and J. Boissonade, Acc. Chem. Res. 9, 438 (1976).

${ }^{23}$ J. Boissonade and P. De Kepper, J. Phys. Chem. 84, 501 (1980).

${ }^{24}$ P. De Kepper, I. R. Epstein, and K. Kustin, J. Am. Chem. Soc. 103, 6121 (1981); 103, 2133 (1981).

${ }^{25}$ R. A. Schmitz, K. R. Graziani, and J. L. Hudson, J. Chem. Phys. 67, 3040 (1977).

${ }^{26}$ J. C. Roux, R. H. Simoyi, and H. L. Swinney, Physica D 8, 257 (1983); R. H. Simoyi, A. Wolf, and H. L. Swinney, Phys. Rev. Lett. 49, 245 (1982); S. K. Scott, Chemical Chaos (Clarendon, Oxford, 1991).

${ }^{27}$ P. W. Davies, P. Blanchedeau, E. Dulos, and P. De Kepper, J. Phys. Chem. A 102, 8236 (1998).

${ }^{28}$ A. Tóth, I. Lagzi, and D. Horváth, J. Phys. Chem. 100, 14838 (1996).

${ }^{29}$ Y. Kuramoto, Chemical Oscillations, Waves, and Turbulence (Springer, Berlin, 1984).

${ }^{30}$ E. Ben-Jacob, Contemp. Phys. 34, 247 (1993).

${ }^{31}$ J. A. Pojman, I. R. Epstein, T. McManus, and K. Showalter, J. Phys. Chem. 95, 1299 (1991).

${ }^{32}$ T. Bánsági Jr., D. Horváth, and A. Tóth, Chem. Phys. Lett. 384, 153 (2004).

${ }^{33}$ D. A. Vasquez and A. De Wit, J. Chem. Phys. 121, 935 (2004).

${ }^{34}$ J. A. Pojman, J. Masere, E. Petretto, M. Rustici, D. S. Huh, M. S. Kim, and V. Volpert, Chaos 12, 56 (2002).

${ }^{35}$ D. Horváth, A. Tóth, and K. Yoshikawa, J. Chem. Phys. 111, 10 (1999).

${ }^{36}$ E. Boga, S. Kádár, G. Peintler, and I. Nagypál, Nature (London) 347, 749 (1990).

${ }^{37}$ M. Pollmann, H. H. Rotermund, G. Ertl, X. Li, and I. G. Kevrekidis, Phys. Rev. Lett. 86, 6038 (2001).

${ }^{38}$ O. Steinbock, A. Tóth, and K. Showalter, Science 267, 868 (1995).

${ }^{39}$ O. Steinbock, P. Kettunen, and K. Showalter, J. Phys. Chem. 100, 18970 (1996).

${ }^{40}$ I. N. Motoike, K. Yoshikawa, Y. Iguchi, and S. Nakata, Phys. Rev. E 63, 036220 (2001).

${ }^{41}$ J. Gorecki, K. Yoshikawa, and Y. Igarashi, J. Phys. Chem. A 107, 1664 (2003).

${ }^{42}$ J. Christoph, P. Strasser, M. Eiswirth, and G. Ertl, Science 284, 291 (1999).

${ }^{43}$ S. Thouvenel-Romans, K. Agladze, and O. Steinbock, J. Am. Chem. Soc. 124, 10292 (2002)

${ }^{44}$ C. Punckt, M. Bölscher, H. H. Rotermund, A. S. Mikhailov, L. Organ, N. Budiansky, J. R. Scully, and J. L. Hudson, Science 305, 1133 (2004).

${ }^{45}$ R. S. Lillie, J. Gen. Physiol. 7, 473 (1925); R. Suzuki, Adv. Biophys. 9 115 (1976); K. Agladze, S. Thouvenel-Romans, and O. Steinbock, Phys. Chem. Chem. Phys. 3, 1326 (2001).

${ }^{46}$ Z. Noszticzius, W. Horsthemke, W. D. McCormick, H. L. Swinney, and W. Y. Tam, Nature (London) 329, 619 (1987).

${ }^{47}$ J. D. Dockery, J. P. Keener, and J. J. Tyson, Physica D 30, 177 (1988); J. M. Flesselles, A. Belmonte, and V. Gáspár, J. Chem. Soc., Faraday Trans. 94, 851 (1998).

${ }^{48}$ P. J. Heaney and A. M. Davis, Science 269, 1562 (1995).

${ }^{49}$ E. Raub and A. Schall, Z. Metallkd. 30, 149 (1938); I. Kristev and M. Nikolova, J. Appl. Electrochem. 16, 875 (2005).

${ }^{50}$ A. Winfree, Science 175, 634 (1972).

${ }^{51}$ A. M. Turing, Philos. Trans. R. Soc. London 237, 37 (1952).

${ }^{52}$ V. Castets, E. Dulos, J. Boissonade, and P. De Kepper, Phys. Rev. Lett. 64, 2953 (1990).

${ }^{53}$ Q. Ouyang and H. L. Swinney, Nature (London) 352, 610 (1991); Q. Ouyang, V. Castets, J. Boissonade, J. C. Roux, P. De Kepper, and H. L. Swinney, J. Chem. Phys. 95, 351 (1991).

${ }^{54}$ M. Dolnik, I. Berenstein, A. M. Zhabotinsky, and I. R. Epstein, Phys. Rev. Lett. 87, 238301 (2001).

${ }^{55}$ V. Gáspár, G. Bazsa, and M. T. Beck, Z. Phys. Chem. (Leipzig) 264, 43 (1983); M. Jinguji, M. Ishihara, and T. Nakazawa, J. Phys. Chem. 96, 4279 (1992).

${ }^{56}$ L. Kuhnert, K. I. Agladze, and V. I. Krinsky, Nature (London) 337, 244 (1989).

${ }^{57}$ O. Steinbock, V. Zykov, and S. C. Müller, Nature (London) 366, 322 (1993).

${ }^{58}$ T. Sakurai, E. Mihaliuk, F. Chirila, and K. Showalter, Science 296, 2009 (2002). 
${ }^{59}$ V. Petrov, Q. Ouyang, and H. L. Swinney, Nature (London) 388, 655 (1997).

${ }^{60}$ B. Marts, A. Hagberg, E. Meron, and A. L. Lin, Phys. Rev. Lett. 93, 108305 (2004).

${ }^{61}$ O. Steinbock, P. Kettunen, and K. Showalter, Science 269, 1857 (1995)

${ }^{62}$ X. Li, I. G. Kevrekidis, M. Pollmann, A. G. Papathanasiou, and H. H. Rotermund, Chaos 12, 190 (2002).
${ }^{63}$ B. T. Ginn, B. Steinbock, M. Kahveci, and O. Steinbock, J. Phys. Chem. A 108, 1325 (2004).

${ }^{64}$ B. T. Ginn and O. Steinbock, Phys. Rev. Lett. 93, 158301 (2004).

${ }^{65}$ V. K. Vanag and I. R. Epstein, Phys. Rev. Lett. 87, 228301 (2001).

${ }^{66}$ V. K. Vanag and I. R. Epstein, Science 294, 835 (2001).

${ }^{67}$ V. K. Vanag and I. R. Epstein, Phys. Rev. Lett. 90, 098301 (2003). 